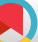

\title{
Nutrition and Cardio-Metabolic Risk Factors: Findings from 20 Years of the Tehran Lipid and Glucose Study
}

\author{
Firoozeh Hosseini-Esfahani ${ }^{1}$, Somaye Hosseinpour-Niazi ${ }^{1}$, Golaleh Asghari ${ }^{1,{ }^{*}}$, Zahra Bahadoran ${ }^{1}$, \\ Nazanin Moslehi ${ }^{1}$, Mahdieh Golzarand ${ }^{1}$, Hanieh-Sadat Ejtahed ${ }^{1}$, Parvin Mirmiran ${ }^{1,{ }^{* *}}$ and Fereidoun \\ Azizi $^{2}$ \\ ${ }^{1}$ Nutrition and Endocrine Research Center, Research Institute for Endocrine Sciences, Shahid Beheshti University of Medical Sciences, Tehran, Iran \\ ${ }^{2}$ Endocrine Research Center, Research Institute for Endocrine Sciences, Shahid Beheshti University of Medical Sciences, Tehran, Iran \\ "Corresponding author: Nutrition and Endocrine Research Center, Research Institute for Endocrine Sciences, Shahid Beheshti University of Medical Sciences, Tehran, Iran. \\ Email: asghari@endocrine.ac.ir

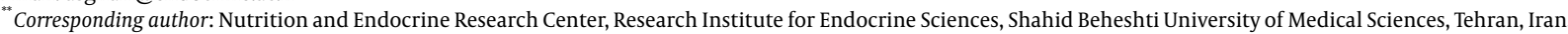 \\ Email: parvin.mirmiran@gmail.com
}

Received 2018 September 30; Revised 2018 October 03; Accepted 2018 October 07.

\section{Abstract}

Context: Genetic and environmental factors contribute to the incidence of metabolic syndrome (MetS). This study aimed to review all findings of studies conducted in framework of the Tehran lipid and glucose study (TLGS) regarding the association of dietary factors with cardio-metabolic risk factors.

Evidence Acquisition: All English-language studies were searched using PubMed and Scopus databases from 2000 to 2017. Finally, 105 relevant papers were included in this review.

Results: Whole grains, legumes, nuts and healthy dietary patterns (DPs) reduced risk of MetS, while white rice, salty/sweet snacks increased this. The western DP had a significant interaction with APOC3, APOA1 and MC4R polymorphisms in relation to MetS. After 6.5 years of follow-up, odds of reaching menarche $\leq 12$ years was significantly higher in girls with higher intakes of milk, calcium, magnesium, and phosphorous. Among children and adolescents, higher adherence to the dietary approaches to stop hypertension (DASH)-style diet decreased the risk of abdominal obesity, whereas increased adherence to the western DP could contribute to general and abdominal obesity. A three-year follow-up of adult participants showed that higher intakes of phytochemical-rich foods were inversely related to development of insulin resistance. Higher adherence to the healthy DPs was associated with the reduced risk of hyperlipidemia and hypertention. Nutrition interventions postponed rise in the prevalence of MetS. The DASH diet resulted in weight reduction compared to control diet.

Conclusions: Higher adherence to healthy food choices was associated with reduced odds of MetS, abdominal obesity, dyslipidemia and hypertension. The western DP accentuated the association of polymorphisms with MetS.

Keywords: Cardiovascular Risk Factors, Diet, Nutrients, Tehran Lipid and Glucose Study, Metabolic Syndrome

\section{Context}

Metabolic syndrome (MetS), is a complex metabolic disorder including abdominal obesity, impaired glucose homeostasis, dyslipidemia, and hypertension, all of which can lead to cardiovascular disease (CVD) and diabetes; its prevalence is fast increasing over the last two decades (1-4). Genetics, sedentary lifestyles and nutrition transition with an accelerating tendency to a westernized diet are considered important factors contributing to the incidence of MetS (5-7). Fast foods with high energy density and higher amounts of fat, saturated fatty acids (SFA) and sodium along with carbonated soft drinks lead to weight gain and increased prevalence of cardio-metabolic risk factors $(2,8$,
9). Also increasing fast food consumption is associated with lower diet quality (9). Higher intakes of fiber and phytochemical rich foods including fruits and vegetables, legumes, nuts and whole grains are related to lower risk of cardio-metabolic risk factors (10). Understanding the overall effects of diets through extracting and defining dietary patterns (DPs) elucidates associations of dietary factors and cardiovascular risk factors (11). The Mediterranean diet (Med) and dietary approach to stop hypertension (DASH) style diet, as models of healthy patterns, have been proposed to play a favorable role in the prevention of CVD (1, 7).

Individuals however are not affected equally by un- 
healthy DPs, some being more sensitive to the harmful effects than others. This heterogeneity may reflect complex interactions between genetic susceptibilities and environmental factors, particularly diet in which some dietary factors modulate the association of polymorphisms and MetS, emphasizing the importance of gene-diet interaction studies $(3,5,12)$.

Tehran lipid and glucose study (TLGS) is an ongoing study initiated in 1999 with a representative sample of 15005 individuals aged $\geq 3$ years, recruited from among residents of district 13 of Tehran, the capital of Iran(13). This prospective study provides an opportunity to study different aspects of cardio-metabolic risk factors in this MiddleEastern population. The aim of this study was to review all findings of studies conducted within the framework of the TLGS regarding the association of dietary factors (nutrients, food groups and dietary patterns) with cardiometabolic risk factors, MetS and its components in different age groups.

\section{Methods}

All English-language studies, from 2000 to 2017, investigating cross-sectional or prospective associations of dietary patterns or food components with development of metabolic disorders in the framework of the TLGS, were searched using PubMed, Scopus, and Embase databases. A structured search strategy using a combination of keywords (diet, nutrition, metabolic syndrome, obesity, insulin resistance, lipids, blood pressure, hypertension, Tehran lipid and glucose study) were conducted to identify records in each database. Nutrigenetic studies conducted in the TLGS were also searched using combination of following key words: Diet, nutrition, gene or polymorphism, Tehran lipid and glucose study. Furthermore, studies focusing on polycystic ovary syndrome and other metabolic disorders in women were also included in this systematic review. Finally, 105 relevant papers were included in this review; 51 papers described the association of dietary factors with the risk of MetS and its components. Associations of nutrition and obesity were described in 58 papers; potential effects of diet and development of insulin resistance were explained in 7 articles, and three focused on nutrition and women's health. Interactions of dietary factors with common polymorphisms of obesity, dyslipidemia and MetS in TLGS population were investigated in 4 papers.

\subsection{Nutrition and Metabolic Syndrome}

2.1.1. Food Groups

Dairy consumption was associated with $18 \%$ reduced risk of MetS in adults (14), although these products did not reduce the risk in adolescents (15). Whole-grain consumption reduced risk of MetS by $32 \%$ in adults (16). Legume consumption more than 3 servings per week reduced risk of MetS by $32 \%$ (17). Nuts and dried fruits consumed $>15.5 \mathrm{~g} / \mathrm{d}$ reduced the risk of MetS by 35\% in adolescents (18).

Fast food consumption had undesirable effects on MetS. The associations of fast food consumption with the occurrence of MetS were more pronounced in younger adults ( $<30$ years) (2). White rice had undesirable effects on the incidence of MetS (19). Energy-dense nutrient-poor solid snacks (both salty and sweet) and sugar sweetened beverages (SSB) were associated with a $>50 \%$ increased incidence of MetS in children, adolescents and adults (Figure 1) (20-23).

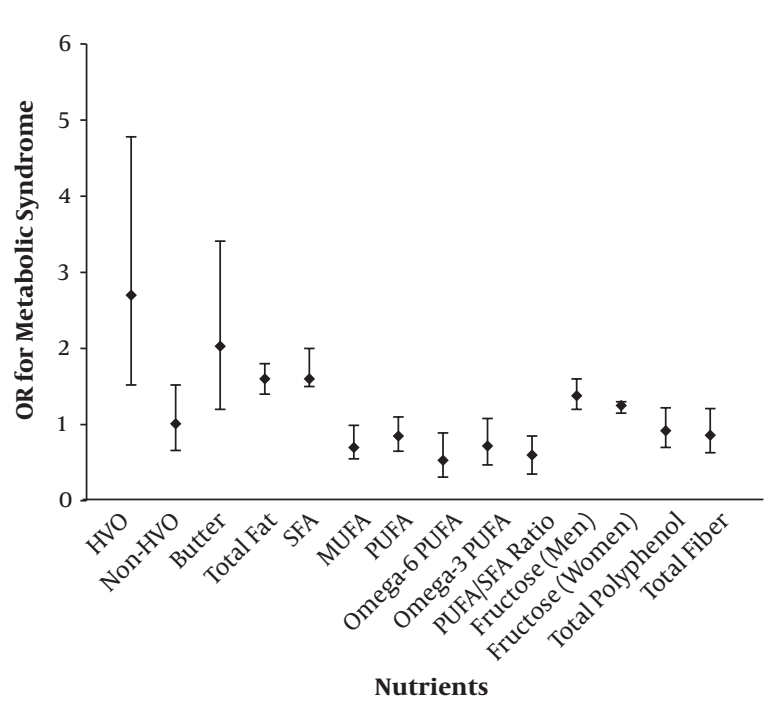

Figure 1. The association of dietary nutrients and metabolic syndrome: Tehran lipid and glucose study. Odds ratios (ORs) were estimated for highest category vs lowest category by adjusted logistic regression analysis. Abbreviations: HVO, hydrogenated vegetable oil; MUFA, Mono-unsaturated fatty acids; PUFA, poly-unsaturated fatty acids; SFA, saturated fatty acids.

\subsubsection{Nutrients}

Low carbohydrate diet (LCD) scores were associated with a decreased risk of MetS in adults (24), although this score had no association with incidence of MetS in children and adolescents (25). Total dietary fiber, solubleand insoluble fiber, fruit fiber, cereal fiber and legume fiber were negatively associated with MetS (26). Higher intake of magnesium consumption reduced risk of MetS in obese participants $(\beta=-0.014, \mathrm{P}<0.05)$ (27). Subclasses of polyphenols such as flavonoids were associated with a lower prevalence of MetS among adults (OR: 0.25, CI: 0.19 - 0.34 in highest vs lowest category) (28). Alpha-linolenic acid (ALA) consumption was inversely associated with the 
MetS, irrespective of the background consumption of n-6 poly-unsaturated fatty acids (PUFAs) in adults (29). Dietary antioxidants had favorable effects on metabolic disorders (30).

Fructose had an association with increased risk of MetS in adults (31). Total fat, especially saturated fat (SFA), Hydrogenated vegetable oils and butter were associated with a higher risk of MetS in adults $(32,33)$. Fatty acids, except PUFA and MUFA consumption, increased the risk of MetS (34); SFA consumption was positively associated with the prevalence of MetS, independent of total dietary fat, MUFA and PUFA consumption (Figure 2) (4).

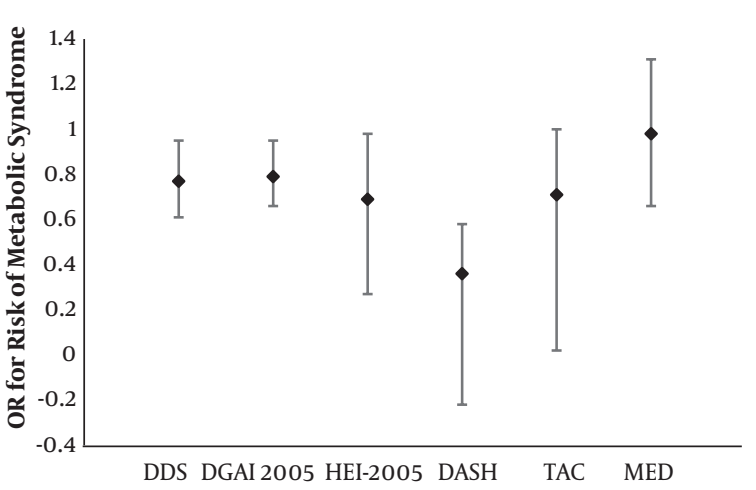

Figure 2. The association of different dietary patterns and metabolic syndrome: Tehran lipid and glucose study. Odds ratios (ORs) were estimated for highest category vs lowest category by adjusted logistic regression analysis. Abbreviations: DASH, dietary approach to stop hypertension; DDS, dietary diversity score; DGAI 2005, dietary guidelines for Americans adherence index 2005 ; HEI-2005, healthy eating index, MED, mediterranean diet; TAC, total antioxidant capacity.

\subsubsection{Dietary Patterns}

Higher adherence to DASH diet and the dietary guidelines for Americans adherence index (DGAI)-2005 reduced risk of MetS $(1,35,36)$. Mediterranean diet score (MDS) was not associated with MetS incidence (7). Dietary diversity score (DDS) had an inverse association with MetS. (37). Whole grains, multiple types of vegetables and fruits, yoghurt and ice cream had protective effects against MetS (38). Adherence to common nutritional targets of public health (total fat, SFA, fiber, fruit and vegetable) had inversely associated with MetS risk factors in Tehranian adults (39). Increased fat consumption and overall unhealthy dietary patterns were associated with MetS (Figure 3) (40).

\subsection{Nutritional Intervention}

Nutrition interventions based on the therapeutic lifestyle change diet guidelines did not reduce the risk of MetS(41), although they did delay the rise in the prevalence of MetS and some of its components (42).

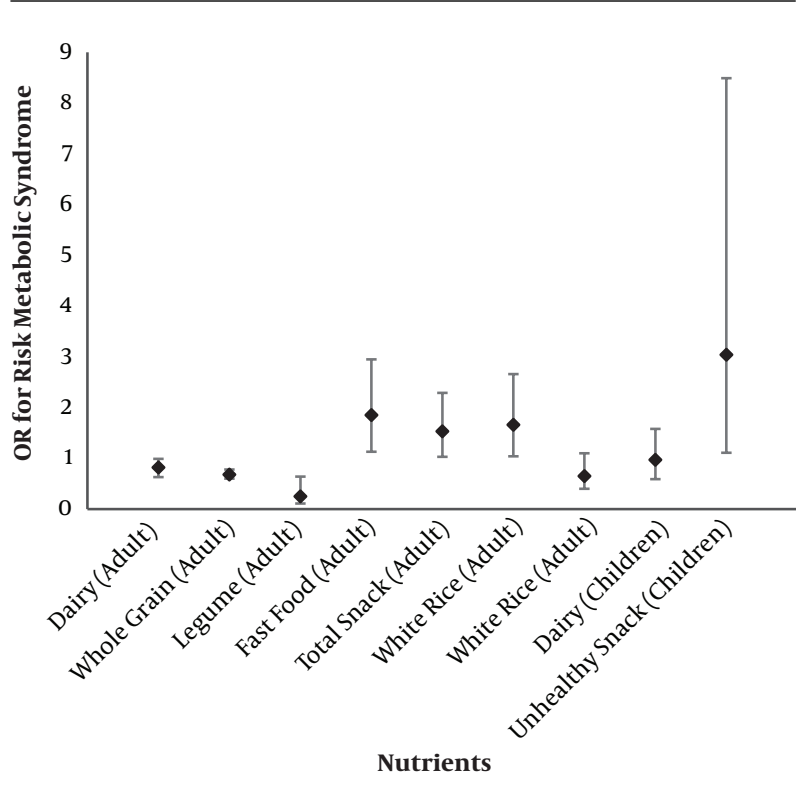

Figure 3. The association of different food group intake and metabolic syndrome: Tehran lipid and glucose study. Odds ratios (ORs) were estimated for highest category vs lowest category by adjusted logistic regression analysis.

\subsection{Nutrigenetic Studies}

The interaction between genetic susceptibilities and dietary factors plays an important role in diet-related polygenic disorders, such as MetS. Western DP scores (loaded heavily on fast food, soft drinks and salty snacks) had a significant interaction with the APOC $33238 \mathrm{C}>\mathrm{G}$ and APOA1 (rs670, -75G $>$ A and rs5069+83C $>$ T) polymorphisms in relation to risk of MetS (11). Compared with other genotype combinations, the combined effect of APOC3/APOA1 $(\mathrm{CC} / \mathrm{GA}+\mathrm{AA} / \mathrm{CT}+\mathrm{TT})$ genotypes showed a further increase in the risk of the MetS in the highest quartile of western DP scores (5).

Among A allele carriers (GA+AA) of MC4R rs12970134, being in the highest quartiles of the western DP score and SFA intake had an increased risk of MetS, compared to those in the lowest quartile (12). A significant interaction was observed between rs12970134 with total fat and iron intake on the risk of abdominal obesity. Moreover there are interactions between omega-3 fatty acids, zinc, salty snacks and ZNT8 variant rs13266634, which may affect the risk of MetS or its components (3).

\subsection{Nutrition and Women's Health}

Timing of menarche occurrence is important because it can affect women's health and reproduction; age at menarche was significantly higher in underweight girls (14.4 \pm 1.1$)$ than normal weight $(13 \pm 1.2)$, overweight $(12.8$ 
$\pm 1.3)$ and obese $(12.8 \pm 1.2)$ ones. BMI and age at menarche were negatively correlated $(\mathrm{r}=-0.13, \mathrm{P}=0.012)(43)$.

A prospective study investigated the association between dairy intakes and timing of menarche among 134 pre-pubertal girls, aged 4 - 12 years. After 6.5 years of followup, odds of reaching menarche $\leq 12$ years was significantly higher in girls with higher intakes of milk (OR $=2.25,95 \%$ CI: 1.03 - 5.05), calcium (OR = 3.20, 95\% CI: 1.39 - 7.42), magnesium $(\mathrm{OR}=2.43,95 \% \mathrm{CI}: 1.12$ - 5.27), and phosphorous (OR =3.37, 95\% CI: 1.44 - 7.87) (44).

Associations between the visceral adiposity index (VAI) DP and cardio-metabolic variables were examined in 53 polycystic ovary syndrome (PCOS) subjects and 167 agematched non-PCOS women. Higher adherence to the pattern was associated with higher triglycerides, the triglycerides/high density lipoprotein cholesterol (HDL-C) ratio and higher odds of visceral adiposity dysfunction. In nonPCOS women with more adherence to the pattern, the odds of hypercholesterolemia, high low density lipoprotein cholesterol (LDL-C), low HDL-C, hyperglycemia and impaired glucose tolerance (IGT)+impaired fasting glucose (IFG) were also higher (45).

Data on a later age at menarche in underweight children with no significant differences in age at menarche between overweight and obese children (43) indicates that BMI is a determinant of menarche although it seems that the association between BMI and menarche may not be linear. The observation of higher risk of early menarche with higher intakes of milk during childhood, (but not cheese and yoghurt) can partly be explained with insulin like growth factor 1(IGF-1) secretion. Stimulation of IGF-1 secretion by bioactive components of milk, i.e. dairy protein, calcium, magnesium, phosphorus increases estrogen production through stimulating adrenal androgen secretion or gonadotropin-releasing hormone $(\mathrm{GnRH})$ by hypothalamic neurons; these bioactive components may be inactive during the processing of milk (44).

\subsection{Nutrition and Obesity}

\subsubsection{Food Groups}

Higher dairy consumption in the TLGS study, both cross-sectional (46) and cohort (14) designs had inverse associations with waist circumference (WC) and obesity in adults. Dairy intake generally showed controversial findings as supported by systematic reviews and metaanalyses. Excessive consumption of high-calorie foods, including sweet drinks and SSB was accompanied with increased risk of overweight and abdominal obesity in two cross-sectional studies in adult participants of the TLGS $(21,47)$, a finding confirmed by most previous studies, in particularly recent systematic reviews that documented a positive association between SSB consumption and obesity indices in children and adults. Findings of these researches lead to exerting tax on SSBs for reducing obesity and chronic diseases.

Dietary intakes of whole grains and vegetables as a part of healthy diet, indicated an association with weight control; in a cross-sectional analysis, adults with higher intakes of whole grain had 10\% lower risk of enlarged WC (16). Similarly, alterations in the consumption of whole grains, vegetables, and added sugars were associated with body weight change, suggesting the effect of food choices on weight control (48). Whereas higher intakes of hydrogenated vegetable oils and advanced glycation end (AGE) products, identified as a part of unhealthy diets are accompanied with increased risk of abdominal obesity $(33,49)$.

Fast foods, which have high energy densities and glycemic loads, could contribute to overweight and obesity. However, in adults no significant difference between 3-year changes of weight with quartile categories of fast food consumption was observed, which may be justified by a wide age of participants ( 19 - 70 years) and a short follow up (3 years) in the study (2). Notably, refined grains (16), legumes (17), fast foods (2), snacks (20), and rice (19) showed no significant association with risk of abdominal obesity or WC values. It is noteworthy that generally these foods had an association with MetS and other chronic diseases, not just weight gain.

Like most other cohorts, among children and adolescents in the TLGS, higher intakes of SSB (OR: 2.49 ; 95\% CI: 1.00 - 6.53) (23), and fast food (OR: 2.58; 95\% CI: 1.01 - 6.61; $\mathrm{P}$ for trend $=0.009$ ) ( 8 ) had increased risk of abdominal obesity. This may be justified by high preference for increased intakes of SSBs and fast food in childhood; fast foods encompass higher percentage of total calorie intake in youth compared to adults. However, no significant relationship was found for abdominal obesity with intakes of dairy (15), energy-dense nutrient-poor solid snacks (22), nuts and dried fruits (50); It seems that children are less tempted to consume dairy, nuts and dried fruits and these foods do not highly contribute to their daily calorie intake.

\subsubsection{Nutrients}

The relationship between macronutrient intakes and body mass index in a group of the TLGS participants was assessed and energy from fat was found to be independently and positively associated with obesity; contradictory to this result, energy from protein and carbohydrate had no association with BMI (51), a finding mostly consistent with results of large population based studies justified by the fact that higher intakes of fat lead to weight gain. However, differences in amounts of protein intakes had a narrow range. 
Cohort studies examined the association of different nutrients, viz. simple sugars (fructose), various types of saturated and unsaturated fats, minerals (calcium and magnesium and some phytochemicals) with overweight and obesity; these studies may provide both consistent or contradictory findings based on the differentiation in study design, population sample size, and other demographic features. In a cross-sectional analysis, TLGS participants with higher intakes of magnesium $(\beta=-0.013, \mathrm{P}=$ 0.006) (27), $\omega-3$ PUFA (OR: 0.52; 95\% CI: 0.35 - 0.75; P trend $=0.014$ ), ALA (OR: 0.54; 95\% CI: $0.37-0.80 ;$ P trend = 0.003) (29), total antioxidant capacity (OR: 0.62; 95\% CI: 0.38 - 0.99; P trend $=0.01$ ) (30), flavonoid (OR: 0.31; 95\% CI: $0.23-0.40 ; \mathrm{P}$ trend $<0.005)(28)$, total fiber $(89.4 \mathrm{~cm}$ vs $91.8 \mathrm{~cm})(26)$, had lower risk of having enlarged WC. Higher intake of fructose (39 in men and $20 \%$ in women) (31), higher $\omega-6 / \omega-3$ ratio (OR: 1.51; 95\% CI: 1.14 - 2.00; P trend = 0.003) (29), and combined effect of total fat and SFA (OR: 1.37; 95\% CI: 1.02 1.83) (4) were accompanied with increased risk of abdominal obesity. Fatty acid composition $(32,34)$, total polyphenol, phenolic acid, stilbene, lignan (28), combined effect of MUFA and SFA and combined effects of PUFA and SFA (4), showed no significant associations with risk of abdominal obesity or WC values.

\subsubsection{Dietary Patterns}

Today, a method commonly used in cohort studies worldwide for evaluation of diet-chronic disease associations is DPs approach, priori or posteriori. The TLGS study aimed to demonstrate the main DPs in an appropriate population sample of Iranians of various demographic subgroups and investigated the association of these patterns with weight changes and obesity. All obesity indices had an increasing trend across quintiles of the traditional DP, which is rich in refined carbohydrates, whole grain, starchy vegetables, other vegetables, red and refined meat, saturated/trans-fat, and egg (52); furthermore, increased adherence to the western DP contributed to general and abdominal obesity (53). However, DDS (37), DGAI-2005 (36), or the Med diet (23) showed no significant association with risk of abdominal obesity or WC values.

In adolescents, BMI was negatively related with breakfast energy percentage in girls $(\mathrm{r}=-0.18, \mathrm{P}<0.01)$ and positively related with lunch energy percentage in both sexes ( $\mathrm{r}=0.16, \mathrm{P}<0.05$ in boys and $\mathrm{r}=0.22, \mathrm{P}<0.01$ in girls $)$ (54). Moreover, evaluation of the relationship between diet quality scores HEI-2005 and HEI-2010, with risk of obesity in Tehranian adolescents revealed that participants in the highest quartile of HEI-2010 compared to those in the lowest had lower risk of general and central obesity (55); however, these indices were not successful in predicting BMI and WC in Iranian adults after 6.7 years of follow-up (56).
No significant relationship of HEI-2005 score (57) and LCD score (25) with abdominal obesity was found among children and adolescents; however, higher adherence to DASHstyle diet decreased the risk of abdominal obesity(OR: 0.35; 95\% CI: 0.14 - 0.89; P trend = 0.047) (1).

Intakes of phytochemical-rich foods, high dietary phytochemical index, and the total antioxidant capacity diet had favorable effects on prevention of weight gain $(30,58)$. There was a significant association of LCD score with WC$\operatorname{BMI}(\beta=-0.003,95 \% \mathrm{CI}:-0.005,-0.001)(24)$.

\subsubsection{Nutrition Intervention}

In addition to these results, community based lifestyle modifications in Tehranian adults were also effective on the prevalence of abdominal obesity (42). Of course, regarding gender differences in the mean daily intakes of energy and nutrients, these behavioral interventions and nutritional recommendations should be targeted and gender-specific (59). The DASH diet resulted in weight reduction among men (-16 and $-14 \mathrm{~kg}$ ) and women (-16 and -15 $\mathrm{kg}$ ) in comparison to the control diet (35). The educational nutrition program had no effects on obesity and abdominal obesity (41). Moreover, a familial obesity pattern was observed in the TLGS population; therefore, familial interventions seem essential to combat obesity in Iran (60).

In summary, whole grains, dairy, fruits, vegetables, phytochemical-rich foods in the framework of a healthy DP are found to be associated with general and abdominal obesity in children, adolescents, and adults. However, some controversies may exist with previous cohort studies; highlighting the need for more investigations in subgroup analyses and longer follow-up durations.

\subsection{Nutrition and Insulin Resistance}

A three-year follow-up of adult participants showed that higher intakes of phytochemical-rich foods $(\mathrm{OR}=0.48$, $95 \% \mathrm{CI}=0.25-0.93)$, in the last compared to the first quartile, dietary intakes of $\beta$-carotene $(\mathrm{OR}=0.42,95 \% \mathrm{CI}=0.25$ - 0.72) and $\beta$-cryptoxanthin $(\mathrm{OR}=0.51,95 \% \mathrm{CI}=0.30-0.84)$ were inversely related to development of insulin resistance (IR) $(61,62)$. Healthy DP (characterized by higher load of vegetables, fresh and dried fruits, low-fat dairy, vegetable oils, nuts and seeds) score was negatively related to 3-year changes in fasting serum insulin $(\beta=-4.91,95 \% \mathrm{CI}=-9.47$, -0.39) and IR index ( $\beta=-5.99,95 \% \mathrm{CI}=-11.7,-0.23)$ (63); compared to the lowest tertile of healthy pattern score, the highest was accompanied with an $81 \%$ reduced risk of IR $(\mathrm{OR}=0.19,95 \% \mathrm{CI}=0.10-0.36)(64)$. Dietary inflammatory index was not significantly related to risk of IR in our population (65), whereas potential renal acid load $(\mathrm{OR}=2.81,95 \%$ $\mathrm{CI}=1.32-5.97$ ) and net endogenous acid production score 
$(\mathrm{OR}=2.18,95 \% \mathrm{CI}=1.03-4.61)$ were positively related to development of IR (66); compared to the lowest, the highest tertile of dietary insulin load was also significantly associated with increased risk of $\operatorname{IR}(\mathrm{OR}=1.69,95 \% \mathrm{CI}=1.01-2.89)$ (67).

Several dietary indicators, e.g. phytochemical index, healthy dietary pattern score, renal acid load, and insulin load have been identified to be potential factors, which could affect development of IR in the TLGS population.

\subsection{Nutrition and Dyslipidemia}

\subsubsection{Food Groups}

Participants in the highest quartile of whole-grain intake had a significantly lower prevalence of hypertriglyceridemic waist phenotype (22\%) than those in the lowest quartile. Conversely, those in the highest quartile of refined-grain intake had a significantly higher prevalence (2-fold) of the hypertriglyceridemic waist phenotype than did those in the lowest quartile (68). The odds of prevalent hypertriglyceridemia (total cholesterol $\geq 240 \mathrm{mg} / \mathrm{dL}$ ) for participants in the highest quartile of whole grains compared to those in the lowest, was 0.61. Furthermore, subjects in the highest quartile of refined-grain intake had higher chances of being hypercholestrolemic, hypertriglyceridemic, and having high LDL-C. A significant decreasing trend was observed among participants in the top quartile of whole-grain intake for having high-serum triglyceride levels, similar to subjects in the upper quartile of refined-grain intake, who had also higher chances of having high-serum triglyceride levels (triglycerides $\geq$ $150 \mathrm{mg} / \mathrm{dL}$ ) (16). A significant increasing trend was observed for prevalence of high triglyceride concentrations across quartiles of dairy products (14). In another study, multivariate-adjusted odds ratios for the high total cholesterol and high LDL-C across quartiles of fruit and vegetable intakes were 0.82 and 0.79 , respectively (69). Fast food consumption had a positive association with serum triglycerides and negative association HDL-C in middleaged adults (31 - 51 years) (9). The odds of prevalent low HDL-C had increasing trends across increasing categories of SSB consumption (21). Among men, total intakes of fruit and vegetables were inversely associated with 3year changes in triglycerides and total cholesterol. Yellow fruits and vegetables were inversely associated with 3-year changes of total cholesterol and HDL-C. Dietary intakes of green fruit and vegetable were inversely associated with triglycerides levels and triglycerides/HDL-C ratio. Moreover, white fruit and vegetable intakes were related to lower abdominal fat gain and inversely associated with total cholesterol changes. Among women, higher consumption of red/purple fruit and vegetable was inversely related to 3-year changes of total cholesterol. Consumption of orange fruits and vegetables had significant inverse associations with 3-year changes of total cholesterol (70). Higher intake of hydrogenated vegetable oils was positively associated with 3-year changes of serum triglycerides. Furthermore, intake of butter was positively associated with serum triglycerides and negatively associated with HDL-C levels (33). A negative association was observed between allium vegetable intakes and 6 year changes of triglyceride levels (71).

\subsubsection{Nutrients}

There were no significant differences in low HDL-C across dietary fructose quartiles in men and women (31). Findings of another study showed that participants in the highest quartile of fat consumption had increased odds of having high serum triglycerides (40). Serum total cholesterol and HDL-C were increased in participants with higher intake of SFA $(32,72)$. Intakes of $\omega$-3 PUFA, eicosapentaenoic acid (EPA) plus docosahexaenoic acid (DHA), and ALA were inversely associated with high serum triacylglycerol concentrations. In addition, a higher EPA+DHA intake was associated with a $34 \%$ lower concentration of high serum triacylglycerol in participants with a lower $\omega-6$ PUFA intake and a $28 \%$ lower concentration in subjects with a higher $\omega-6$ PUFA intake, compared to those with lower EPA+DHA intake (29). Serum LDL-C and triglyceride levels were positively associated with SFA intake, whereas LDL-C was inversely associated with mono-unsaturated fatty acid (MUFA). HDL-C level was inversely associated with SFA and PUFA intake and the positive association with MUFA and the ratio of PUFA to SFA. The LDL/HDL-C ratio was negatively associated with the ratio of PUFA to SFA ratio (34). A combination of high SFA intake and low MUFA intake was associated with high serum triglyceride concentrations (4). Serum HDL-C was associated with total protein intake in men and women (73). Dietary magnesium was inversely associated with serum triglycerides, while copper intake was positively associated with $\operatorname{HDL}-\mathrm{C}(27,74)$. Dietary total antioxidant capacity were negatively associated with triglycerides, and positively associated with HDL-C levels (30). Those in highest quartile of dietary flavonoid intakes had 65\% lower risk of hypertriglyceridemia and 33\% lower risk of low HDL-C, compared to those in the lowest quartile. In addition, subjects in the highest quartile of dietary lignan intakes had higher odds of (33\%) having hypertriglyceridemia (28). Dietary potential renal acid load was associated with serum triglycerides, HDL-C. In addition, protein to potassium ratio was associated with serum HDL-C and triglycerides (75). The highest quartile of protein intake was inversely associated with 3-year changes in total cholesterol and HDL-C levels in men, compared to 
the lowest quartile. Dietary protein to carbohydrate ratio in men was associated with 3-year changes in serum triglycerides and total cholesterol. In women, dietary protein and protein to carbohydrate ratio showed no significant relation with lipid profile changes (76). Participants in the highest compared to the lowest quartile of dietary fiber and phytochemical-rich foods decreased risk of incident hypertriglyceridemia by $42 \%$ after 3 years of followup. In addition, higher dietary sodium to potassium ratios compared to lower ratios increased the risk of hypertriglyceridemia by $63 \%$ (77). Med score was not associated with components of MetS (23). There was no significant association between serum nitric-oxide (NOx) and the incidence of hypertriglyceridemic-waist in men; however, serum NOx in women increased by $46 \%$ the risk of incident hypertriglyceridemic-waist (78).

\subsubsection{Dietary Patterns}

Higher adherence to the DGAI was associated with a 31\% decreased risk of low HDL-C prevalence(36). Participants in highest quartile of DDS, compared with those in the lowest ones had 16\% lower risk of high serum triglycerides (37). The risk of having hypertriglyceridaemia decreased with increasing quartiles of the diversity score for whole grains. The odds of having hypercholesterolaemia and high LDL-C decreased with increasing quartiles of the diversity score for vegetables. The probability of having hypercholesterolaemia and high LDL-C decreased with quartiles of the DDS (79). Men in the highest quartile of the HEI-2005 score had significantly lower triglyceride changes than those in the lowest quartile, although, there was no such relation in women (80). LCD score was negatively associated with triglycerides among adults (24). There were positive correlations between dietary carbohydrate and serum triglycerides, cholesterol intake and serum LDL-C (81). Higher dietary glycemic index was associated with higher triglyceride concentrations and lower HDL-C among obese individuals (BMI > 30) (82). Compared with those in the upper quartiles, participants in the lower quartile of dietary phytochemical index had a 36\% lower risk of elevated serum triglycerides (10). Dietary intakes of AGE products were not associated with prevalence of high triglycerides and low HDL-C (49).

An inverse association was observed between dietary phytochemical index and 3 years changes of total cholesterol, triglycerides, HDL-C, and non-HDL-C in men, while this index had no association in women (83). In addition, risk for incidence of hypertriglyceridemic waist phenotype in participants with the highest compared to the lowest dietary phytochemical index was significantly reduced (84). Higher adherence to the traditional DP score was associated with changes in HDL-C level during 4.7 years of follow-up. However, there was no significant association between western DP scores and changes in serum HDL-C (85).

\subsubsection{Nutrition and Dyslipidemia in Children and Adolescents}

Adolescents in the highest HEI-2005 category had 38\% lower odds of prevalence high triglycerides and 37\% lower risk of low HDL-C compared with those in the lowest category (57). Among adolescents, serum triglycerides decreased with increasing the quartiles of energy intake from PUFA. Moreover, serum HDL-C level decreased according to the quartiles of percent of energy intake from PUFA (86). In adolescents aged 10 - 19 years, dietary intake of dairy products was not associated with prevalence of high triglycerides and low HDL-C (15). Among children and adolescents, aged 10 - 19 years the concentration of triglycerides significantly decreased across the quartiles of nuts and dried fruit intakes (50). Among adolescents those in the lowest quartile of LCD score, compared with those in the highest quartile had odds ratios of 0.55 and 0.49 in the incidence of high triglycerides and low HDL-C, respectively (25). Among children and adolescents aged 6 - 18 years, dietary intakes of SSBs had no association with high triglycerides and low HDL-C after 3 years of follow-up (23). Children and adolescents in highest quartile of fast food intake had a 2.8-fold higher risk of incident high triglycerides than those in the lowest intakes after 3 years of follow-up (8). After 3 years of follow-up in children and adolescents, higher adherence to the dietary approaches to stop hypertension style had no association with incidence of high triglycerides and low HDL-C (1). There was no significant association of dietary intakes of salty and sweetened snacks with incidence of high triglycerides and low HDL-C among children and adolescents after 3 years of follow-up (22).

To sum up, higher adherence to prudent DPs such as DGAI and HEI-2005 might improve dyslipidemia. In addition, increasing dietary diversity was associated with reduced hypercholesterolemia risk and decreased serum triglycerides. Besides, higher compliance with the LCD and dietary phytochemical index were associated with lower risk of hyperlipidemia. It seems that prudent dietary patterns have beneficial effects on lipid profiles due to high content of bioactive compounds, increased dietary diversity, and low dietary glycemic index.

Most of the studies have focused on the relationship between dietary fatty acids intake and dyslipidemia. Other food components such as magnesium, polyphenols, and fiber were also associated with dyslipidemia; this favorable effect on the lipid profile might pertain to the increased total anti-oxidant capacity and decreased dietary acid load, both of which had a significant association with lipid profile. 
Adherence to the prudent dietary pattern such as HEI, DASH, and LCD might ameliorate or protect children and adolescents against dyslipidemia. Higher adherence to the healthy DP is associated with higher intakes of prudent foods, containing high amounts of antioxidants, magnesium, dietary fiber, fruits, and vegetables, all of which are beneficial for lipid hemostasis. Consumption of low glycemic index foods may improve insulin sensitivity and decrease cardiovascular risk factors in children and adolescents. The utility of diet quality indices in assessing eating behaviors of children and adolescents in relation to dyslipidemia may provide a valuable tool for monitoring and preventive strategies in public health education and intervention programs.

\subsection{Nutrition and Hypertension}

\subsubsection{Food Groups}

In a cross-sectional study on 827 adults, whole-grain consumption had a significant inverse association with high blood pressure (BP) (OR: 0.84, 95\% CI: 0.73 - 0.99) (16). Subjects in the highest quartile of white rice intake had higher BP in compared to those in the lowest quartile of intake (19). Consumption of protein food groups had no association with the 3-year incidence of hypertension (87). In a case-control study of 240 adults, legume consumption reduced systolic but not diastolic BP (17). Consumption of dairy, high-fat dairy, non-fermented dairy and milk had inverse association with BP and hypertension in adults $(14,88)$ an association not observed in adolescents (15). In a prospective study conducted on 1087 adults, total dairy intake increased and decreased 3-year risk of hypertension incidence in men and women, respectively (89). Nuts, dried fruits and dietary magnesium showed nonsignificant association with $\mathrm{BP}(18,27)$.

In two prospective studies, fast food consumption had no association with incidence of hypertension after a 3year follow-up in both adolescents and adults $(2,8)$. There was no association between salty and sweet snacks and 3-year incidence of hypertension in adults (90); however, salty- but not sweet snacks had a significant inverse association with elevated BP in children and adolescents (22). SSB intake whether in children, adolescents or adults was associated with higher risk of elevated $\mathrm{BP}(21,23)$. The highest tertile of nitrate-containing vegetables reduced 3-year incidence of hypertension by $37 \%$, compared to the lowest tertile (91). Intakes of Allium vegetables had no relationship with risk of hypertension after a 6-year follow-up (71).

\subsubsection{Nutrients}

In an observational study, dietary fructose consumption showed a significant relationship with BP and systolic
BP in men and women, respectively (31). Results of a crosssectional study on 2537 adults showed that BP had no association with dietary protein intake and animal-to-plant protein ratio (73). In two cross-sectional studies, no relationships of BP with consumption of PUFA, MUFA, SFA, PUFA to SFA ratio, oleic acid and linoleic acid was found (32, 34). However, high dietary SFA increased risk of high BP independent of total fat and MUFA; on the contrary, high SFA among subjects with high PUFA intake decreased the risk of high BP by $9 \%$ (4). There was no significant association between hydrogenated, non-hydrogenated vegetable oils and butter and 3-year changes in systolic and diastolic BP (33). Dietary nitrite was inversely associated with 6-year risk of hypertension (OR: 0.58, 95\% CI: 0.33 - 0.98) (92).

\subsubsection{Dietary Patterns}

The highest quartile of DDS was associated with 15\% lower risk of high blood pressure (BP), compared to the lowest quartile of DDS (OR: 0.85, 95\% CI: 0.58 - 1.13, P trend $=0.03$ ) (37). DGAI-2005 was inversely associated with elevated BP (OR: 0.76, 95\% CI: 0.70 - 0.93) (36). Healthy DP was inversely associated with systolic BP(OR: 0.74, 95\% CI: 0.56 - 0.97) but not diastolic BP (93). There was no significant relationship between western DP and BP. In another study on 2241 adults (23), adherence to the Med diet had no association with 3-year changes in systolic BP and diastolic BP. In a cross-sectional study of 706 adolescents, HEI-2005 had no association with systolic- or diastolic BP (57). Asghari et al. (1) have indicated that compared to the lowest quartile of DASH, the highest quartile of DASH-style diet reduced 3-year risk of elevated $\mathrm{BP}$ by $30 \%$. In a prospective study conducted on 2044 adults, LCD score had a negative association with high $\mathrm{BP}(\beta=-0.001, \mathrm{P}=0.04)$ after 3.6-years (24), although it had no relationship with BP in adolescents (25). In a cross-sectional study dietary phytochemical index showed no significant association with BP (10) although the highest quartile of this index was associated with the lower odds ratio 3-year incidence of hypertension (OR: 0.52, 95\% CI: 0.32 - 0.84) (94). Dietary total antioxidant capacity and dietary flavonoid intake have a significant inverse association with elevated $\mathrm{BP}(28,30)$. In a cross-sectional study (49), there was no relationship between AGEs products and elevated BP. Renal acid load $(\beta=$ $0.06, \mathrm{P}=0.006)$ and protein to potassium ratio $(\beta=0.02, \mathrm{P}$ $=0.04)$ were positively associated with diastolic BP, but not systolic BP(75).

\subsubsection{Nutrition Intervention}

Results of a longitudinal study reported that community-based education for lifestyle intervention had no effect on BP over a 3.6-year follow up (42). 
Overall, whole grains, legumes, dairy and vegetables decreased risk of high BP, while fast foods and SSB elevated the risk of hypertension. Among nutrients high SFA intake increased risk of high BP, while dietary nitrite was inversely associated with risk of hypertension. Adherence to healthy DPs was inversely associated with high BP.

\section{Conclusions}

Some micro- and macronutrients, healthy DPs and healthy food groups were associated with reduced odds of cardio-metabolic risk factors. Unhealthy food groups like SSB and fast food increased cardiovascular risk factors. Unhealthy dietary patterns modified the association of polymorphisms with Mets.

It is recommended to investigate time trends of conforming dietary factors in accordance with dietary guidelines and their relations to MetS, abdominal obesity, dyslipidemia and hypertension for designing guidelines and implementing strategies aimed at disease prevention.

\section{Acknowledgments}

The authors wish to acknowledge Ms. Niloofar Shiva for critical editing of English grammar and syntax of this manuscript.

\section{Footnotes}

Authors' Contribution: Firoozeh Hosseini-Esfahani, Somaye Hosseinpour-Niazi, Golaleh Asghari, Zahra Bahadoran, Nazanin Moslehi, Mahdieh Golzarand, Hanieh-Sadat Ejtahed designed the study and interpreted the data, and drafting the manuscript; Fereidoun Azizi and Parvin Mirmiran supervised the study, Fereidoun Azizi critically revised the manuscript for important intellectual content and final approval of the version to be published.

Financial Disclosure: The authors declare that they have no competing interests.

Funding/Support: The study was supported by Research Institute for Endocrine Sciences, Iran.

\section{References}

1. Asghari G, Yuzbashian E, Mirmiran P, Hooshmand F, Najafi R, Azizi F. Dietary approaches to stop hypertension (DASH) dietary pattern is associated with reduced incidence of metabolic syndrome in children and adolescents. J Pediatr. 2016;174:178-184 e1. doi: 10.1016/j.jpeds.2016.03.077. [PubMed: 27156186].

2. Bahadoran Z, Mirmiran P, Hosseini-Esfahani F, Azizi F. Fast food consumption and the risk of metabolic syndrome after 3-years of followup: Tehran lipid and glucose study. Eur J Clin Nutr. 2013;67(12):1303-9. doi: 10.1038/ejcn.2013.217. [PubMed: 24193228].
3. Hosseini-Esfahani F, Mirmiran P, Koochakpoor G, Daneshpour MS, Guity K, Azizi F. Some dietary factors can modulate the effect of the zinc transporters 8 polymorphism on the risk of metabolic syndrome. Sci Rep. 2017;7(1):1649. doi: 10.1038/s41598-017-01762-9. [PubMed: 28490771]. [PubMed Central: PMC5431973].

4. Hosseinpour-Niazi S, Mirmiran P, Fallah-ghohroudi A, Azizi F. Combined effect of unsaturated fatty acids and saturated fatty acids on the metabolic syndrome: Tehran lipid and glucose study. J Health Popul Nutr. 2015;33:5. doi: 10.1186/s41043-015-0015-z. [PubMed: 26825310]. [PubMed Central: PMC5026005].

5. Hosseini-Esfahani F, Mirmiran P, Daneshpour MS, Mehrabi Y, Hedayati M, Soheilian-Khorzoghi M, et al. Dietary patterns interact with APOA1/APOC3 polymorphisms to alter the risk of the metabolic syndrome: The Tehran lipid and glucose study. Br J Nutr. 2015;113(4):64453. doi: 10.1017/S0007114514003687. [PubMed: 25653052].

6. Hosseinpour-Niazi S, Mirmiran P, Sohrab G, Hosseini-Esfahani F, Azizi F. Inverse association between fruit, legume, and cereal fiber and the risk of metabolic syndrome: Tehran lipid and glucose study. Diabetes Res Clin Pract. 2011;94(2):276-83. doi: 10.1016/j.diabres.2011.07.020. [PubMed: 21856031].

7. Mirmiran P, Moslehi N, Mahmoudof H, Sadeghi M, Azizi F. A longitudinal study of adherence to the mediterranean dietary pattern and metabolic syndrome in a non-mediterranean population. Int J Endocrinol Metab. 2015;13(3). e26128. doi: 10.5812/ijem.26128v2. [PubMed: 26425127]. [PubMed Central: PMC4584365].

8. Asghari G, Yuzbashian E, Mirmiran P, Mahmoodi B, Azizi F. Fast food intake increases the incidence of metabolic syndrome in children and adolescents: Tehran lipid and glucose study. PLoS One. 2015;10(10). e0139641. doi: 10.1371/journal.pone.0139641. [PubMed: 26447855]. [PubMed Central: PMC4598125].

9. Bahadoran Z, Mirmiran P, Golzarand M, Hosseini-Esfahani F, Azizi F. Fast food consumption in Iranian adults; dietary intake and cardiovascular risk factors: Tehran lipid and glucose study. Arch Iran Med. 2012;15(6):346-51. [PubMed: 22642243].

10. Bahadoran Z, Golzarand M, Mirmiran P, Saadati N, Azizi F. The association of dietary phytochemical index and cardiometabolic risk factors in adults: Tehran lipid and glucose study. J Hum Nutr Diet. 2013;26 Suppl 1:145-53. doi: 10.1111/jhn.12048. [PubMed: 23581519].

11. Hosseini-Esfahani F, Mirmiran P, Daneshpour MS, Mehrabi Y, Hedayati $\mathrm{M}$, Zarkesh $\mathrm{M}$, et al. Western dietary pattern interaction with APOC3 polymorphism in the risk of metabolic syndrome: Tehran lipid and glucose study. J Nutrigenet Nutrigenomics. 2014;7(2):105-17. doi: 10.1159/000365445. [PubMed: 25301527].

12. Koochakpoor G, Daneshpour MS, Mirmiran P, Hosseini SA, HosseiniEsfahani F, Sedaghatikhayat B, et al. The effect of interaction between melanocortin-4 receptor polymorphism and dietary factors on the risk of metabolic syndrome. Nutr Metab (Lond). 2016;13:35. doi: 10.1186/s12986-016-0092-z. [PubMed: 27186233]. [PubMed Central: PMC4867980].

13. Azizi F, Ghanbarian A, Momenan AA, Hadaegh F, Mirmiran P, Hedayati $M$, et al. Prevention of non-communicable disease in a population in nutrition transition: Tehran lipid and glucose study phase ii. Trials. 2009;10:5. doi: 10.1186/1745-6215-10-5. [PubMed: 19166627]. [PubMed Central: PMC2656492].

14. Azadbakht L, Mirmiran P, Esmaillzadeh A, Azizi F. Dairy consumption is inversely associated with the prevalence of the metabolic syndrome in Tehranian adults. Am J Clin Nutr. 2005;82(3):523-30. doi: 10.1093/ajcn.82.3.523. [PubMed: 16155263].

15. Ghotboddin Mohammadi S, Mirmiran P, Bahadoran Z, Mehrabi Y, Azizi F. The association of dairy intake with metabolic syndrome and its components in adolescents: Tehran lipid and glucose study. Int J Endocrinol Metab. 2015;13(3). e25201. doi: 10.5812/ijem.25201v2. [PubMed: 26425126]. [PubMed Central: PMC4584419]. 
16. Esmaillzadeh A, Mirmiran P, Azizi F. Whole-grain consumption and the metabolic syndrome: A favorable association in Tehranian adults. Eur J Clin Nutr. 2005;59(3):353-62. doi: 10.1038/sj.ejcn.1602080. [PubMed: 15536473].

17. Hosseinpour-Niazi S, Mirmiran P, Amiri Z, Hosseini-Esfahani F, Shakeri N, Azizi F. Legume intake is inversely associated with metabolic syndrome in adults. Arch Iran Med. 2012;15(9):538-44. [PubMed: 22924370].

18. Mirmiran P, Ghotboddin Mohammadi S, Bahadoran Z, Azizi F. Study of nuts and dried fruits consumption in adolescents in relation to risk of metabolic syndrome and its components: Tehran lipid and glucose study. Int J Nutr Food Sci. 2016;5(1-2):8-13. doi: 10.11648/j.ijnfs.s.2016050102.12.

19. Bahadoran Z, Mirmiran P, Delshad H, Azizi F. White rice consumption is a risk factor for metabolic syndrome in Tehrani adults: A prospective approach in Tehran lipid and glucose study. Arch Iran Med. 2014;17(6):435-40. [PubMed: 24916530].

20. Mirmiran P, Bahadoran Z, Delshad H, Azizi F. Effects of energydense nutrient-poor snacks on the incidence of metabolic syndrome: A prospective approach in Tehran lipid and glucose study. Nutrition. 2014;30(5):538-43. doi: 10.1016/j.nut.2013.09.014. [PubMed: 24508464].

21. Ejtahed HS, Bahadoran Z, Mirmiran P, Azizi F. Sugar-sweetened beverage consumption is associated with metabolic syndrome in Iranian adults: Tehran lipid and glucose study. Endocrinol Metab (Seoul). 2015;30(3):334-42. doi: 10.3803/EnM.2015.30.3.334. [PubMed: 26435135]. [PubMed Central: PMC4595359].

22. Asghari G, Yuzbashian E, Mirmiran P, Bahadoran Z, Azizi F. Prediction of metabolic syndrome by a high intake of energy-dense nutrient-poor snacks in Iranian children and adolescents. Pediatr Res. 2016;79(5):697-704. doi:10.1038/pr.2015.270. [PubMed: 26717004].

23. Mirmiran P, Yuzbashian E, Asghari G, Hosseinpour-Niazi S, Azizi F. Consumption of sugar sweetened beverage is associated with incidence of metabolic syndrome in Tehranian children and adolescents. Nutr Metab (Lond). 2015;12:25. doi: 10.1186/s12986-015-0021-6. [PubMed: 26225136]. [PubMed Central: PMC4518610].

24. Mirmiran P, Asghari G, Farhadnejad H, Eslamian G, Hosseini-Esfahani F, Azizi F. Low carbohydrate diet is associated with reduced risk of metabolic syndrome in Tehranian adults. Int $J$ Food Sci Nutr. 2017;68(3):358-65. doi: 10.1080/09637486.2016.1242119. [PubMed: 27718762].

25. Eslamian G, Mirmiran P, Asghari G, Hosseini-Esfahani F, Yuzbashian E, Azizi F. Low carbohydrate diet score does not predict metabolic syndrome in children and adolescents: Tehran lipid and glucose study. Arch Iran Med. 2014;17(6):417-22. [PubMed: 24916527].

26. Hosseinpour-Niazi S, Mirmiran P, Mirzaei S, Azizi F. Cereal, fruit and vegetable fibre intake and the risk of the metabolic syndrome: A prospective study in the Tehran lipid and glucose study. J Hum Nutr Diet. 2015;28(3):236-45. doi: 10.1111/jhn.12242. [PubMed: 24890325].

27. Mirmiran P, Shab-Bidar S, Hosseini-Esfahani F, Asghari G, Hosseinpour-Niazi S, Azizi F. Magnesium intake and prevalence of metabolic syndrome in adults: Tehran lipid and glucose study. Public Health Nutr. 2012;15(4):693-701. doi: 10.1017/S1368980011002941. [PubMed: 22217579].

28. Sohrab G, Hosseinpour-Niazi S, Hejazi J, Yuzbashian E, Mirmiran P, Azizi F. Dietary polyphenols and metabolic syndrome among Iranian adults. Int J Food Sci Nutr. 2013;64(6):661-7. doi: 10.3109/09637486.2013.787397. [PubMed: 23607642].

29. Mirmiran P, Hosseinpour-Niazi S, Naderi Z, Bahadoran Z, Sadeghi M, Azizi F. Association between interaction and ratio of omega-3 and omega- 6 polyunsaturated fatty acid and the metabolic syndrome in adults. Nutrition. 2012;28(9):856-63. doi: 10.1016/j.nut.2011.11.031. [PubMed: 22459553].

30. Bahadoran Z, Golzarand M, Mirmiran P, Shiva N, Azizi F. Dietary total antioxidant capacity and the occurrence of metabolic syndrome and its components after a 3-year follow-up in adults: Tehran lipid and glu- cose study. Nutr Metab (Lond). 2012;9(1):70. doi: 10.1186/1743-7075-9-70. [PubMed: 22849424]. [PubMed Central: PMC3556123].

31. Hosseini-Esfahani F, Bahadoran Z, Mirmiran P, Hosseinpour-Niazi S, Hosseinpanah F, Azizi F. Dietary fructose and risk of metabolic syndrome in adults: Tehran lipid and glucose study. Nutr Metab (Lond) 2011;8(1):50. doi: 10.1186/1743-7075-8-50. [PubMed: 21749680]. [PubMed Central: PMC3154855].

32. Hekmatdoost A, Mirmiran P, Hosseini-Esfahani F, Azizi F. Dietary fatty acid composition and metabolic syndrome in Tehranian adults. Nutrition. 2011;27(10):1002-7. doi: 10.1016/j.nut.2010.11.004. [PubMed: 21907897].

33. Hosseinpour-Niazi S, Mirmiran P, Hosseini-Esfahani F, Azizi F. Is the metabolic syndrome inversely associates with butter, nonhydrogenated- and hydrogenated-vegetable oils consumption: Tehran lipid and glucose study. Diabetes Res Clin Pract. 2016;112:20-9. doi: 10.1016/j.diabres.2015.11.008. [PubMed: 26655020].

34. Shab-Bidar S, Hosseini-Esfahani F, Mirmiran P, Hosseinpour-Niazi S, Azizi F. Metabolic syndrome profiles, obesity measures and intake of dietary fatty acids in adults: Tehran lipid and glucose study.J Hum Nutr Diet. 2014;27 Suppl 2:98-108. doi: 10.1111/jhn.12117. [PubMed: 23731333].

35. Azadbakht L, Mirmiran P, Esmaillzadeh A, Azizi T, Azizi F. Beneficial effects of a dietary approaches to stop hypertension eating plan on features of the metabolic syndrome. Diabetes Care. 2005;28(12):282331. [PubMed: 16306540].

36. Hosseini-Esfahani F, Jessri M, Mirmiran P, Bastan S, Azizi F. Adherence to dietary recommendations and risk of metabolic syndrome: Tehran lipid and glucose study. Metabolism. 2010;59(12):1833-42. doi: 10.1016/j.metabol.2010.06.013. [PubMed: 20667561].

37. Azadbakht L, Mirmiran P, Azizi F. Dietary diversity score is favorably associated with the metabolic syndrome in Tehranian adults. Int Obes (Lond). 2005;29(11):1361-7. doi: 10.1038/sj.ijo.0803029. [PubMed: 16116493].

38. Cheraghi Z, Mirmiran P, Mansournia MA, Moslehi N, Khalili D, Nedjat $\mathrm{S}$. The association between nutritional exposures and metabolic syndrome in the Tehran lipid and glucose study (TLGS): A cohort study. Public Health. 2016;140:163-71. doi: 10.1016/j.puhe.2016.07.003. [PubMed: 27498945].

39. Hosseini-Esfahani F, Jessri M, Mirmiran P, Sadeghi M, Azizi F. Does the diet of Tehranian adults ensure compliance with nutritional targets? Observations from the Tehran lipid and glucose study. Public Health Nutr. 2011;14(9):1539-48. doi: 10.1017/S1368980011000711. [PubMed: 21557877].

40. Mirmiran P, Hekmatdoost A, Azizi F. Metabolic syndrome is associated with adherence to an unhealthy diet. Diabetes Care. 2007;30(9). e93. doi: 10.2337/dc06-1928. [PubMed:17726183].

41. Ramezankhani A, Mirmiran P, Azizi F. Effect of nutritional intervention on the prevalence of metabolic syndrome and heart disease risk factors in urban Tehran (Tehran lipid and glucose study). East Mediterr Health J. 2011;17(6):501-8. [PubMed: 21796968].

42. Azizi F, Mirmiran P, Momenan AA, Hadaegh F, Habibi Moeini A, Hosseini $\mathrm{F}$, et al. The effect of community-based education for lifestyle intervention on the prevalence of metabolic syndrome and its components: tehran lipid and glucose study. Int J Endocrinol Metab. 2013;11(3):145-53. doi: 10.5812/ijem.5443. [PubMed: 24348586]. [PubMed Central: PMC3860109].

43. Ramezani Tehrani F, Mirmiran P, Gholami R, Moslehi N, Azizi F. Factors influencing menarcheal age: Results from the cohort of tehran lipid and glucose study. Int I Endocrinol Metab. 2014;12(3) e16130. doi: 10.5812/ijem.16130. [PubMed: 25237321]. [PubMed Central: PMC4166004].

44. Ramezani Tehrani F, Moslehi N, Asghari G, Gholami R, Mirmiran P, Azizi F. Intake of dairy products, calcium, magnesium, and phosphorus in childhood and age at menarche in the Tehran lipid and glucose study. PLoS One. 2013;8(2). e57696. doi: 10.1371/journal.pone.0057696. [PubMed: 23451261]. [PubMed Central: PMC3581542]. 
45. Ehsani B, Moslehi N, Mirmiran P, Ramezani Tehrani F, Tahmasebinejad Z,Azizi F.A visceral adiposity index-related dietary pattern and the cardiometabolic profiles in women with polycystic ovary syndrome. Clin Nutr. 2016;35(5):1181-7. doi: 10.1016/j.clnu.2015.10.007. [PubMed: 26699405].

46. Mirmiran P, Esmaillzadeh A, Azizi F. Dairy consumption and body mass index: An inverse relationship. Int J Obes (Lond). 2005;29(1):11521. doi: 10.1038/sj.ijo.0802838. [PubMed: 15534616].

47. Mirmiran P, Ejtahed HS, Bahadoran Z, Bastan S, Azizi F. Sugarsweetened beverage consumption and risk of general and abdominal obesity in iranian adults: Tehran lipid and glucose study. Iran J Public Health. 2015;44(11):1535-43. [PubMed: 26744712]. [PubMed Central: PMC4703234].

48. Hosseini Esfahani F, Ejtahed HS, Mirmiran P, Delshad H, Azizi F Alterations in food group intakes and subsequent weight changes in adults: Tehran lipid and glucose study. Int J Endocrinol Metab. 2014;12(3). e17236. doi: 10.5812/ijem.17236. [PubMed: 25237324]. [PubMed Central: PMC4166206].

49. Angoorani P, Ejtahed HS, Mirmiran P, Mirzaei S, Azizi F. Dietary consumption of advanced glycation end products and risk of metabolic syndrome. Int I Food Sci Nutr. 2016;67(2):170-6. doi: 10.3109/09637486.2015.1137889. [PubMed: 26850840].

50. Mirmiran P. Study of nuts and dried fruits consumption in adolescents in relation to risk of metabolic syndrome and its components: Tehran lipid and glucose study. Int J Nutr Food Sci. 2016;5(1):8. doi: 10.11648/j.ijnfs.s.2016050102.12.

51. Mirmiran P, Esmaillzadeh A, Azizi F. Diet composition and body mass index in Tehranian adults. Asia Pac J Clin Nutr. 2006;15(2):224-30. [PubMed: 16672207].

52. Sherafat-Kazemzadeh R, Egtesadi S, Mirmiran P, Gohari M, Farahani $\mathrm{SJ}$, Esfahani FH, et al. Dietary patterns by reduced rank regression predicting changes in obesity indices in a cohort study: Tehran lipid and glucose study. Asia Pac J Clin Nutr. 2010;19(1):22-32. [PubMed: 20199984].

53. Hosseini-Esfahani F, Djazaieri SA, Mirmiran P, Mehrabi Y, Azizi F. Which food patterns are predictors of obesity in Tehranian adults? J Nutr Educ Behav. 2012;44(6):564-73. doi: 10.1016/j.jneb.2010.08.004. [PubMed: 21652267].

54. Azizi F, Allahverdian S, Mirmiran P, Rahmani M, Mohammadi F. Dietary factors and body mass index in a group of Iranian adolescents: Tehran lipid and glucose study 2. Int J Vitam Nutr Res. 2001;71(2):123-7. doi: 10.1024/0300-9831.71.2.123. [PubMed: 11339109].

55. Mohseni-Takalloo S, Hosseini-Esfahani F, Mirmiran P, Azizi F. Associations of pre-defined dietary patterns with obesity associated phenotypes in Tehranian adolescents. Nutrients. 2016;8(8). doi: 10.3390/nu8080505. [PubMed: 27548211]. [PubMed Central: PMC4997418].

56. Asghari G, Mirmiran P, Rashidkhani B, Asghari-Jafarabadi M, Mehran M, Azizi F. The association between diet quality indices and obesity: Tehran lipid and glucose study. Arch Iran Med. 2012;15(10):599-605. [PubMed: 23020534].

57. Mohseni-Takalloo S, Mirmiran P, Hosseini-Esfahani F, Mehrabi Y, Azizi F. Metabolic syndrome and its association with healthy eating index2005 in adolescents: Tehran lipid and glucose study. J Food Nutr Res. 2014;2(4):155-61. doi: 10.12691/jfnr-2-4-4.

58. Mirmiran P, Bahadoran Z, Golzarand M, Shiva N, Azizi F. Association between dietary phytochemical index and 3-year changes in weight, waist circumference and body adiposity index in adults: Tehran lipid and glucose study. Nutr Metab (Lond). 2012;9(1):108. doi: 10.1186/1743 7075-9-108. [PubMed: 23206375]. [PubMed Central: PMC3546027].

59. Mirmiran P, Mohammadi F, Sarbazi N, Allahverdian S, Azizi F. Gender differences in dietary intakes, anthropometrical measurements and biochemical indices in an urban adult population: The Tehran lipid and glucose study. Nutr Metab Cardiovasc Dis. 2003;13(2):64-71 [PubMed: 12929618].

60. Mirmiran P, Mirbolooki M, Azizi F. Familial clustering of obesity and the role of nutrition: Tehran lipid and glucose study. Int J Obes Relat Metab Disord. 2002;26(12):1617-22. doi: 10.1038/sj.ijo.0802120. [PubMed: 12461678].

61. Bahadoran Z, Mirmiran P, Tohidi M, Azizi F. Dietary phytochemical index and the risk of insulin resistance and beta-cell dysfunction: A prospective approach in Tehran lipid and glucose study. Int J Food Sci Nutr. 2015;66(8):950-5. doi: 10.3109/09637486.2015.1111867. [PubMed: 26600067].

62. Mirmiran P, Khalili Moghadam S, Bahadoran Z, Tohidi M, Azizi F. Association of dietary carotenoids and the incidence of insulin resistance in adults: Tehran lipid and glucose study. Nutr Diet. 2016;73(2):162-8. doi: 10.1111/1747-0080.12244.

63. Doostvandi T, Bahadoran Z, Mozaffari-Khosravi H, Mirmiran P, Azizi F. Food intake patterns are associated with the risk of impaired glucose and insulin homeostasis: A prospective approach in the Tehran lipid and glucose study. Public Health Nutr. 2016;19(13):2467-74. doi: 10.1017/S1368980016000616. [PubMed: 27087273].

64. Doostvandi T, Bahadoran Z, Mozaffari-Khosravi H, Tahmasebinejad Z, Mirmiran P, Azizi F. The association of dietary patterns and the incidence of insulin resistance after a 3-year follow-up: Tehran lipid and glucose study. Asia Pac J Clin Nutr. 2017;26(3):531-8. doi: 10.6133/apjcn.032016.12. [PubMed: 28429920].

65. Moslehi N, Ehsani B, Mirmiran P, Shivappa N, Tohidi M, Hebert JR, et al. Inflammatory properties of diet and glucose-insulin homeostasis in a cohort of Iranian adults. Nutrients. 2016;8(11). doi: 10.3390/nu8110735. [PubMed: 27869717]. [PubMed Central: PMC5133119].

66. Moghadam SK, Bahadoran Z, Mirmiran P, Tohidi M, Azizi F. Association between dietary acid load and insulin resistance: Tehran lipid and glucose study. Prev Nutr Food Sci. 2016;21(2):104-9. doi: 10.3746/pnf.2016.21.2.104. [PubMed: 27390726]. [PubMed Central: PMC4935236].

67. Mirmiran P, Esfandiari S, Bahadoran Z, Tohidi M, Azizi F. Dietary insulin load and insulin index are associated with the risk of insulin resistance: A prospective approach in tehran lipid and glucose study.J Diabetes Metab Disord. 2015;15:23. doi: 10.1186/s40200-016-02475. [PubMed: 27446819]. [PubMed Central: PMC4955203].

68. Esmaillzadeh A, Mirmiran P, Azizi F. Whole-grain intake and the prevalence of hypertriglyceridemic waist phenotype in Tehranian adults. Am J Clin Nutr. 2005;81(1):55-63. doi: 10.1093/ajcn/81.1.55. [PubMed: 15640460].

69. Mirmiran P, Noori N, Zavareh MB, Azizi F. Fruit and vegetable consumption and risk factors for cardiovascular disease. Metabolism. 2009;58(4):460-8. doi: 10.1016/j.metabol.2008.11.002. [PubMed: 19303965].

70. Mirmiran P, Bahadoran Z, Moslehi N, Bastan S, Azizi F. Colors of fruits and vegetables and 3-year changes of cardiometabolic risk factors in adults: Tehran lipid and glucose study. Eur J Clin Nutr. 2015;69(11):12159. doi:10.1038/ejcn.2015.49. [PubMed: 25852026].

71. Bahadoran Z, Mirmiran P, Momenan AA, Azizi F. Allium vegetable intakes and the incidence of cardiovascular disease, hypertension, chronic kidney disease, and type 2 diabetes in adults: A longitudinal follow-up study. J Hypertens. 2017;35(9):1909-16. doi: 10.1097/HJH.0000000000001356. [PubMed: 28319598].

72. Mirmiran P, Ramezankhani A, Azizi F. Combined effects of saturated fat and cholesterol intakes on serum lipids: Tehran lipid and glucose study. Nutrition. 2009;25(5):526-31. doi: 10.1016/j.nut.2008.11.018. [PubMed: 19121920].

73. Mirmiran P, Hajifaraji M, Bahadoran Z, Sarvghadi F, Azizi F. Dietary protein intake is associated with favorable cardiometabolic risk factors in adults: Tehran lipid and glucose study. Nutr Res. 2012;32(3):16976. doi:10.1016/j.nutres.2012.01.003. [PubMed: 22464803].

74. Shab-Bidar S, Hosseini-Esfahani F, Mirmiran P, Mehran M, Azizi F. Dietary intakes of zinc and copper and cardiovascular risk factors in Tehranian adults: Tehran lipid and glucose study. Nutr Diet. 2013;70(3):218-26. doi: 10.1111/1747-0080.12026. 
75. Bahadoran Z, Mirmiran P, Khosravi H, Azizi F. Associations between dietary acid-base load and cardiometabolic risk factors in adults: The Tehran lipid and glucose study. Endocrinol Metab (Seoul). 2015;30(2):201-7. doi: 10.3803/EnM.2015.30.2.201. [PubMed: 25433661]. [PubMed Central: PMC4508265].

76. Bahadoran Z, Mirmiran P, Hosseini-Esfahabni F, Sadeghi M, Azizi F. Dietary protein, protein to carbohydrate ratio and subsequent changes in lipid profile after a 3-year follow-up: Tehran lipid and glucose study. Iran J Public Health. 2013;42(11):1232-41. [PubMed: 26171335]. [PubMed Central: PMC4499064].

77. Mirmiran P, Bahadoran Z, Mirzaei S, Azizi F. Dietary intake, changes in lipid parameters and the risk of hypertriglyceridemia: A prospective approach in the Tehran lipid and glucose study. Int J Vitam Nutr Res. 2014;84(5-6):269-76. doi: 10.1024/0300-9831/a000213. [PubMed: 26255548].

78. Bahadoran Z, Mirmiran P, Ghasemi A, Azizi F. Serum nitric oxide metabolites are associated with the risk of hypertriglyceridemicwaist phenotype in women: Tehran lipid and glucose study. Nitric $O x$ ide.2015;50:52-7. doi: 10.1016/j.niox.2015.08.002. [PubMed:26284308].

79. Azadbakht L, Mirmiran P, Esmaillzadeh A, Azizi F. Dietary diversity score and cardiovascular risk factors in Tehranian adults. Public Health Nutr. 2006;9(6):728-36. [PubMed: 16925878].

80. Asghari G, Mirmiran P, Hosseni-Esfahani F, Nazeri P, Mehran M, Azizi F. Dietary quality among Tehranian adults in relation to lipid profile: Findings from the Tehran lipid and glucose study.J Health Popul Nutr. 2013;31(1):37-48. [PubMed: 23617203]. [PubMed Central: PMC3702357].

81. Azadbakht L, Mirmiranr R, Azizi F. Predictors of cardiovascular risk factors in Tehranian adults: Diet and lifestyle. East Mediterr Health J. 2006;12(1-2):88-97. [PubMed: 17037225].

82. Hosseinpour-Niazi S, Sohrab G, Asghari G, Mirmiran P, Moslehi N, Azizi F. Dietary glycemic index, glycemic load, and cardiovascular disease risk factors: Tehran lipid and glucose study. Arch Iran Med. 2013;16(7):401-7. [PubMed: 23808777].

83. Golzarand M, Mirmiran P, Bahadoran Z, Alamdari S, Azizi F. Dietary phytochemical index and subsequent changes of lipid profile: $A$ 3-year follow-up in Tehran lipid and glucose study in Iran. ARYA Atheroscler. 2014;10(4):203-10. [PubMed: 25258636]. [PubMed Central: PMC4173317].

84. Mottaghi A, Bahadoran Z, Mirmiran P, Mirzaei S, Azizi F. Is dietary phytochemical index in association with the occurrence of hypertriglyceridemic waist phenotype and changes in lipid accumulation product index? A prospective approach in Tehran lipid and glucose study. Int J Pharmacog Phytochem Res. 2015;7(1):16-21.
85. Mirmiran P, Bahadoran Z, Vakili AZ, Azizi F. Western dietary pattern increases risk of cardiovascular disease in Iranian adults: A prospective population-based study. Appl Physiol Nutr Metab. 2017;42(3):32632. doi: 10.1139/apnm-2016-0508. [PubMed: 28177742].

86. Mohseni-Takalloo S, Mirmiran P, Hosseini-Esfahani F, Azizi F. Dietary fat intake and its relationship with serum lipid profiles in Tehranian adolescents. J Food Nutr Res. 2014;2(6):330-4. doi: 10.12691/jfnr-2-6-10.

87. Golzarand M, Bahadoran Z, Mirmiran P, Azizi F. Protein foods group and 3-year incidence of hypertension: A prospective study from Tehran lipid and glucose study. J Ren Nutr. 2016;26(4):219-25. doi: 10.1053/j.jrn.2016.01.017. [PubMed: 26908191].

88. Mirmiran P, Golzarand M, Bahadoran Z, Mirzaei S, Azizi F. Highfat dairy is inversely associated with the risk of hypertension inadults: Tehran lipid and glucose study. Int Dairy J. 2015;43:22-6. doi: 10.1016/j.idairyj.2014.11.003.

89. Mirmiran P, Golzarand M, Bahadoran Z, Ataee M, Azizi F. Paradoxical association of dairy intake between men and women with the incidence of hypertension: A three-year follow up in Tehran lipid and glucose study. Nutr Diet. 2016;73(2):153-61. doi:10.1111/1747-0080.12228.

90. Mottaghi A, Hojjat P, Mirmiran P, Azizi F. The effect of unhealthy snacks on incidence of hypertension in adults during 3 years follows up: Tehran lipid and glucose study. Bull Env Pharmacol Life Sci. 2015;4:84-6.

91. Golzarand M, Bahadoran Z, Mirmiran P, Zadeh-Vakili A, Azizi F. Consumption of nitrate-containing vegetables is inversely associated with hypertension in adults: A prospective investigation from the Tehran lipid and glucose study. J Nephrol. 2016;29(3):377-84. doi: 10.1007/s40620-015-0229-6. [PubMed: 26335410].

92. Bahadoran Z, Mirmiran P, Ghasemi A, Carlstrom M, Azizi F, Hadaegh F. Association between dietary intakes of nitrate and nitrite and the risk of hypertension and chronic kidney disease: Tehran lipid and glucose study. Nutrients. 2016;8(12). doi: 10.3390/nu8120811. [PubMed: 28009811]. [PubMed Central: PMC5188466].

93. Mirmiran P, Hosseini Esfahani F, Mottaghi A, Azizi F. Longitudinal study of association between dietary patterns and hypertension in adults: Tehran lipid and glucose study. Sch JApp Med Sci. 2015;3(1B):6571.

94. Golzarand M, Bahadoran Z, Mirmiran P, Sadeghian-Sharif S, Azizi F. Dietary phytochemical index is inversely associated with the occurrence of hypertension in adults: A 3-year follow-up (the Tehran lipid and glucose study). Eur J Clin Nutr. 2015;69(3):392-8. doi 10.1038/ejcn.2014.233. [PubMed: 25387902]. 